\title{
Correction to: APASL clinical practice guideline on hepatitis B reactivation related to the use of immunosuppressive therapy
}

\author{
George Lau ${ }^{1,2}$ (D) Ming-Lung $\mathrm{Yu}^{3} \cdot$ Grace Wong $^{4} \cdot$ Alexander Thompson $^{5} \cdot$ Hasmik Ghazinian $^{6} \cdot$ Jin-Lin Hou ${ }^{7}$. \\ Teerha Piratvisuth $^{8}$. Ji-Dong Jia ${ }^{9} \cdot$ Masashi Mizokami ${ }^{10}$. Gregory Cheng ${ }^{2,11}$. Guo-Feng Chen ${ }^{12}$. Zhen-Wen Liu ${ }^{13}$. \\ Oidov Baatarkhuu ${ }^{14}$. Ann Lii Cheng ${ }^{15}$. Woon Leung $\mathrm{Ng}^{16}$. Patrick Lau ${ }^{1}$. Tony Mok ${ }^{17}$. Jer-Ming Chang ${ }^{18}$. \\ Saeed Hamid ${ }^{19}$. A. Kadir Dokmeci ${ }^{20} \cdot$ Rino A. Gani ${ }^{21}$. Diana A. Payawal ${ }^{22}$. Pierce Chow ${ }^{23}$. Joong-Won Park ${ }^{24}$. \\ Simone I. Strasser ${ }^{25} \cdot$ Rosmawaiti Mohamed $^{26} \cdot$ Khin Maung Win $^{27} \cdot$ Tawesak Tanwandee $^{28} \cdot$ Shiv Kumar Sarin $^{29}$. \\ Masao Omata ${ }^{30,31}$
}

Received: 8 January 2022 / Accepted: 8 January 2022 / Published online: 25 January 2022

(c) Asian Pacific Association for the Study of the Liver 2022

\section{Correction to: \\ Hepatology International (2021) 15:1031-1048 \\ https://doi.org/10.1007/s12072-021-10239-x}

Unfortunately, the first and last name of the Co-author, Tawesak Tanwandee has been interchanged in the original publication of the article. The correct first name should read as "Tawesak" and the last name should read as "Tanwandee" respectively.

The original article can be found online at https://doi.org/10.1007/ s12072-021-10239-x.

George Lau

gkklau@hnhmgl.com; gkklau@netvigator.com

$\triangle$ Ming-Lung Yu

fish6069@gmail.com

1 Humanity and Health Clinical Trial Center, Humanity and Health Medical Group, Hong Kong SAR, China

2 The Fifth Medical Center of Chinese, PLA General Hospital, Beijing 100039, China

3 Hepatitis Center and Hepatobiliary Division, Department of Internal Medicine, Kaohsiung Medical University Hospital, Kaohsiung Medical University, No. 100, Tz-You 1st Rd, Chinese Taipei, Kaohsiung, Taiwan

4 Department of Medicine and Therapeutics, The Chinese University of Hong Kong, Hong Kong SAR, China

5 St Vincent's Hospital, Melbourne, VIC, Australia

6 Department of Hepatology, Nork Clinical Hospital of Infectious Diseases, Yerevan, Armenia

7 Department of Infectious Diseases, Institute of Hepatology, Nanfang Hospital, Southern Medical University, Guangzhou, China
Publisher's Note Springer Nature remains neutral with regard to jurisdictional claims in published maps and institutional affiliations.
8 Department of Medicine, NKC Institute of Gastroenterology and Hepatology, Songklanagarind Hospital, Prince of Songkla University, Hat Yai, Thailand

9 Liver Research Center, Beijing Friendship Hospital, Beijing, China

10 Genome Medical Science Project, National Center for Global Health and Medicine, Ichikawa, Japan

11 Faculty of Health Science, Macau University, Macau SAR, China

12 Department of Liver Diseases, Fifth Medical Center of Chinese, PLA General Hospital, Beijing, China

13 Research Center for Liver Transplantation, Fifth Medical Center of Chinese PLA General Hospital, Beijing, China

14 Department of Infectious Diseases, Mongolian National University of Medical Sciences, Ulaanbaatar, Mongolia

15 Department of Internal Medicine, National Taiwan University Hospital, Taipei, Taiwan

16 Department of Medicine, United Christian Hospital, Hong Kong SAR, China 
17 Department of Clinical Oncology, State Key Laboratory of South China, Chinese University of Hong Kong, Hong Kong SAR, China

18 Division of Nephrology, Kaohsiung Medical University Hospital, Kaohsiung Medical University, Kaohsiung, Taiwan

19 Department of Medicine, Aga Khan University and Hospital, Stadium Road, Karachi 74800, Pakistan

20 Department of Gastroenterology, Ankara University School of Medicine, Ankara, Turkey

21 Liver Transplantation Team, Ciptomangunkusumo Hospital, Jakarta, Indonesia

22 Department of Medicine, Cardinal Santos Medical Center, Mandaluyong, Metro, Manila, Philippines

23 Department of Hepatopancreatobiliary and Transplant Surgery, Singapore General Hospital, Singapore, Singapore

24 Center for Liver Cancer, Research Institute and Hospital, National Cancer Center, Goyang, Korea
25 AW Morrow Gastroenterology and Liver Centre, Royal Prince Alfred Hospital, Sydney, Australia

26 Department of Medicine, University Malaya Medical Centre, Kuala Lumpur, Malaysia

27 Yangon Gastroenterology and Liver Centre, Yangon, Myanmar

28 Division of Gastroenterology, Department of Medicine, Faculty of Medicine, Siriraj Hospital, Mahidol University, Bangkok, Thailand

29 Department of Hepatology, Institute of Liver and Biliary Sciences, New Delhi, India

30 Yamanashi Prefectural Central Hospital, 1-1-1 Fujimi, Kofu-shi, Yamanashi 400-8506, Japan

31 The University of Tokyo, 7-3-1 Hongo, Bunkyo-ku, Tokyo 113-8655, Japan 